\title{
On the control of some chaotic systems by using dither
}

\author{
Ömer Morgül * \\ Bilkent University, Dept. of Electrical and Electronics Engineering, 06533 Bilkent, Ankara, Turkey \\ Received 30 March 1999; received in revised form 31 August 1999; accepted 3 September 1999 \\ Communicated by A.P. Fordy
}

\begin{abstract}
In this Letter, we propose the application of dither for controlling chaotic systems in Lur'e form. Dither is a high frequency periodic signal that can be used for stabilization of limit cycles in some type of nonlinear systems. We apply the dither to change some parameters of the system which may determine its behaviour. We also present some simulation results. (C) 1999 Elsevier Science B.V. All rights reserved.
\end{abstract}

PACS: $05.45 . \mathrm{Gg}$

Keywords: Chaos; Dither; Control of chaos; Lur'e systems

\section{Introduction}

Recently, there has been an extensive interest on the study of analysis and control of chaotic behaviour in nonlinear systems. Similar to classical control problems, various problems could be defined for the control of chaotic systems. Some authors define the control problem as targeting trajectories to a desired point, see [1], others as the elimination of multiple basins of attraction, see [2], and yet others as stabilizing unstable periodic orbits, see $[3,4]$, main goal being the suppression of chaos. We note that the literature is quite rich on this subject, and review of various methodologies can be found in $[5,6]$. For additional information and references, see [7-10]. In this Letter we propose a technique which may enable

\footnotetext{
*Fax: 90-312-266 43 07; e-mail: morgul@ee.bilkent.edu.tr
}

us to switch between chaotic and regular (e.g. periodic) trajectories of some chaotic systems.

In this work, we propose the application of a dither signal for the control of a class of chaotic systems, see Fig. 1. Such systems are said to be in Lur'e form and will be explained in the next section. Dither is a high frequency periodic signal, commonly used in the control of nonlinear systems to stabilize limit cycles, see $[11,12]$. When dither is applied as in Fig. 1, it modifies the nonlinearity by sweeping quickly accross the domain of the nonlinear element. In other words, under some weak conditions the dither applied system given in Fig. 1 is equivalent to a similar system without dither provided that the nonlinear block is replaced by an appropriate nonlinearity. If dynamical behaviour of the system depends on some parameters and if these parameters can be effectively changed by the application of dither, then by choosing an appropriate dither it may be possible to control the behaviour of the system. Dither does 


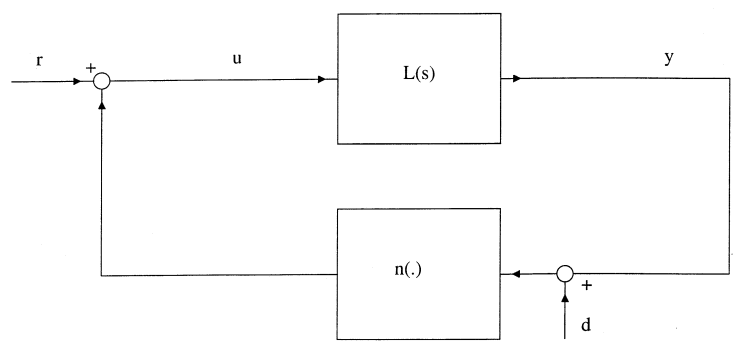

Fig. 1. A Lur'e system with dither.

not depend on signals of the system to be controlled. Hence the application of dither is essentially an open-loop control technique, as opposed to closedloop techniques which requires feedback. We note that open-loop control methods are known to have many limitations and disadvantages; they are sensitive to noise and disturbances, they depend on the model of system and are not robust against parameters mismatch, etc. However, main advantages of open-loop control methods are their simplicity and economy, which makes them attractive for certain applications.

The use of periodic forcing in chaotic systems, mainly to eliminate chaotic behaviour has been investigated before, see e.g. [13-15]. In [14], the Duffing-Holmes equation, which is a special case of the Duffing equation given in (14) has been considered. By changing the coefficient of the cubic term from $p$ to $p(1+\eta \cos \Omega t)$ where $\eta$ is small, it was shown in [14] that for certain values of $\Omega$, it is possible to eliminate chaotic behaviour. In [15] a damped pendulum with DC (i.e. constant) and AC (i.e. sinusoidal) forcing terms is considered. By adding a new sinusoidal forcing term to this system, it was shown in [15] that depending on the frequency it is possible to convert chaotic behaviour into a regular one (i.e. limit cycle). See also [5] for additional references. Note that both of these techniques can be considered as open-loop control techniques in which certain parameters of the system to be controlled are modified periodically. In our method, certain signals of the system to be controlled, namely the output $y$ of the linear block, are modified periodically, see Fig. 1. Besides, while the results of $[14,15]$ depend on the frequency of the perturbation, our results do not, as long as dither frequency is sufficiently high.
It is known that some chaotic systems are either in Lur'e form, or can be converted into it, see [16]. The use of dither to control such chaotic systems has also been suggested in e.g. [17-19]. In [17], some theoretical results have been shown, in [18] some experimental results in an electronic chaotic circuit have been demonstrated and in [19] both theoretical and experimental results have been given. Although our approach shows some similarities with the references cited above, there are some differences as well. In [19], the effect of square-wave, sinusoidal and triangular wave dithers on the output of the nonlinear block were given and then this idea was applied to some chaotic systems. In our work we use only square-wave type dither, but while only symmetric dither was considered in [19] (i.e. $d(t)$ takes constant values $W$ and $-W$ alternately in each half period), we consider a general square-wave dither, see (3). Moreover, we show the effect of the dither signal to the system parameters, and propose a general methodology to change the behaviour of the system to be controlled. The systems we consider are more general than the ones considered in $[17,18]$ and the latter contains mainly some experimental results.

This Letter is organized as follows. In Section 2 we will give an outline of our method. In Section 3 we will present some simulation results. The first two simulations are based on some Lur'e type systems, one of which is the well-known forced Duffing oscillator. The third simulation is based on an unusual forcing of the well-known Van der Pol oscillator, which is not in Lur'e form. This simulation suggests that the proposed technique may be applied to systems not in Lur'e form as well.

\section{Dither control}

We consider the systems given in Fig. 1, which are named after the Soviet scientist Lur'e who investigated them in 1960s. Such systems are composed of a linear dynamical block whose output is fed back to its input via a nonlinear transformation. Here, $L(s)$ represents the transfer function of a linear, time-invariant block, $n(\cdot)$ represents a memoryless nonlinearity, $y$ is the system output, $r(t)$ is an external signal, which could be regarded as a reference input, and $d(t)$ is the dither signal applied to 
the system. Let us assume that the linear block be represented by a transfer function $L(s)=p(s) / q(s)$ where $p(s)$ and $q(s)$ are polynomials in $s$ given as follows:

$$
\begin{aligned}
& p(s)=c_{n-1} s^{n-1}+\ldots+c_{1} s+c_{0}, \\
& q(s)=s^{n}+b_{n-1} s^{n-1}+\ldots+b_{1} s+b_{0} .
\end{aligned}
$$

In (1), all coefficients are assumed to be real constants. The dynamical equation of the system in terms of the output variable $y$ can be given as ( $D$ $\left.=\frac{d}{d t}\right)$ :

$q(D) y+p(D) n(y+d)=p(D) r$.

Main use of dither in Lur'e systems is the possibility of modifying the nonlinearity $n(\cdot)$. Due to its high frequency and periodic nature, a dither signal has the effect of averaging the nonlinearity. Under some weak conditions, the system in Fig. 1 with dither signal $d(t)$ is equivalent to the system in Fig. 1 without dither provided that the nonlinearity $n(\cdot)$ is replaced by a suitable nonlinearity $n_{r}(\cdot)$. The form of equivalent nonlinearity $n_{r}(\cdot)$ depends on dither signal, see e.g. [12,17]. For some simple dither signals, $n_{r}(\cdot)$ in terms of dither parameters can be obtained easily. Then, by using dither parameters, and using (1)-(2), it may be possible to change some parameters of the system, and by using this property it may be possible to control the behaviour of the system to a certain extent. This is the rationale in using dither.

We consider the following piecewise continuous periodic signal as dither:

$d(t)= \begin{cases}\beta_{1} & k T<t<\left(\alpha_{1}+k\right) T, \\ \beta_{2} & \left(\alpha_{1}+k\right) T<t<\left(\alpha_{1}+\alpha_{2}+k\right) T, \\ , \ldots & \ldots, \\ \beta_{l} & \left(\sum_{i=1}^{l-1} \alpha_{i}+k\right) T<t<(k+1) T,\end{cases}$

where $\beta_{i} \in \mathbb{R} ; \alpha_{i}>0$ for $i=1,2, \ldots, l ; \sum_{i=1}^{l} \alpha_{i}=$ 1 and $T>0$. It can be shown that with this dither, if the nonlinearity $n(\cdot)$ satisfies certain smoothness conditions and if the period $T$ is sufficiently small, then the nonlinearity $n(\cdot)$ could be replaced by the following function $n_{r}(y)$ :

$$
\begin{aligned}
n_{r}(y)= & \alpha_{1} n\left(y+\beta_{1}\right)+\alpha_{2} n\left(y+\beta_{2}\right)+\ldots . \\
& +\alpha_{l} n\left(y+\beta_{l}\right) .
\end{aligned}
$$

That is, the system in Fig. 1 with the dither signal $\mathrm{d}(\mathrm{t})$ given by (3) is equivalent to the system in Fig. 1 without dither provided that the nonlinearity $n(\cdot)$ is replaced by $n_{r}($.) given in (4), see e.g. [12,17]. Here the equivalence is in the sense that trajectories of both systems starting from the same initial conditions converge each other. If solutions of both systems are bounded, then this property holds provided that $n(\cdot)$ is (locally) Lipschitz, see [12]. The period $T$ is not important, as long as $1 / T$ is much bigger then the cut-off frequency of the linear block. Hence, $L(s)$ should be low-pass, i.e. $|L(j \omega)| \rightarrow 0$ as $\omega \rightarrow \infty$.

To simplify the computations, let us assume that the nonlinearity $n(\cdot)$ is also a polynomial in $y$ as given below:

$n(y)=a_{m} y^{m}+a_{m-1} y^{m-1}+\ldots+a_{1} y+a_{0}$,

where the coefficients $a_{i}$ are also assumed to be constant. Now consider the dither applied system given in Fig. 1. After the application of dither signal given by (3), by using (4) we find that the nonlinearity $n(y)$ given by (5) changes to the following nonlinearity $n_{r}(y)$ :

$n_{r}(y)=\sum_{k=0}^{m} a_{k} \sum_{i=0}^{k}\left(\begin{array}{l}k \\ i\end{array}\right) \sum_{j=1}^{l} \alpha_{j} \beta_{j}^{(k-i)} y^{i}$.

It can easily be shown that (6) can be written as:

$n_{r}(y)=n(y)+\sum_{i=0}^{m-1} r_{i} y^{i}$,

where the coefficients $r_{i}$ are given as:

$r_{i}=\sum_{k=1}^{m} a_{k}\left(\begin{array}{c}k \\ i\end{array}\right) \sum_{j=1}^{l} \alpha_{j} \beta_{j}^{(k-i)}$.

Hence after the application of dither, dynamical equation of the system becomes:

$q(D) y+p(D) n_{r}(y)=p(D) r$.

From (5) and (7) it follows that $n_{r}(y)=\sum_{j=0}^{j=m} \hat{a}_{j} y^{j}$ where $\hat{a}_{m}=a_{m}$ and $\hat{a}_{j}=a_{j}+r_{j}, 0 \leq j \leq m-1$. In other words, after the application of dither effective nonlinearity has the same form as given by (5) but with different coefficients. Hence if the dynamical behaviour of chaotic system depends on some of these coefficients, we could modify them by the use of dither, and required dither parameters may be found by using (8). We note that the form of (8) 
imposes some constraints on $r_{j}$, hence an arbitrary change in the parameters may not be possible.

We can also rearrange (9) as follows. If we define a new nonlinearity $n_{e}$ as $n_{e}(y)=n_{r}(y)-r_{1} y$, then (9) could be further modified as $\hat{q}(D) y+p(D)$ $n_{e}(y)=p(D) r$, where $\hat{q}(s)$ is defined as: $\hat{q}(s)$ $=s^{n}+\left(b_{n-1}+c_{n-1} r_{1}\right) s^{n-1}+\ldots+\left(b_{1}+c_{1} r_{1}\right) s+$ $\left(b_{0}+c_{0} r_{1}\right)$. In other words, after the application of dither, the system changes to another Lur'e system whose linear part is given by the transfer function $\hat{L}(s)=p(s) / \hat{q}(s)$ and the nonlinear part is given by the nonlinearity $n_{e}(\cdot)$. Hence by choosing dither parameters, we could change some parameters of the system, and by using this degree of freedom we may control its behaviour. If a bifurcation diagram in terms of the parameters of $q(s)$ and/or $n(\cdot)$ is available, then by choosing dither parameters appropriately, we may change the behaviour of the system. We note that due to the structure of $p(s)$ and/or $r_{j}$, it may not be possible to change the parameters of $q(s)$ and/or $n(\cdot)$ arbitrarily. We also note that the constant term $a_{0}$ in (5) changes to $a_{0}+r_{0}$ after the application of dither, see (7), (8). If we do not want to change $a_{0}$, then even if $r_{0} \neq 0$, we could apply an input $r(t)=c_{0} r_{0}$ to cancel the effect of this term.

Let us consider the special cases such as $n(y)=y^{2}$ or $n(y)=y^{3}$, which is frequently encountered in chaotic systems proposed in the literature. For these cases, we assume that dither is given by (3), with $l=2$. When $n(y)=y^{2}$ the coefficients $r_{1}$ and $r_{0}$ are calculated from (8) as:

$r_{1}=2\left(\alpha_{1} \beta_{1}+\left(1-\alpha_{1}\right) \beta_{2}\right)$,

$r_{0}=\alpha_{1} \beta_{1}^{2}+\left(1-\alpha_{1}\right) \beta_{2}^{2}$.

Since $0<\alpha_{1}<1$, we cannot have $r_{0}=0$, but the effect of $r_{0}$ can be eliminated by applying an appropriate constant input $r(t)=c_{0} r_{0}$. By choosing $r_{1}$ appropriately we may modify the polynomial $\hat{q}(s)$. For the case $n(y)=y^{3}$, the coefficients $r_{2}, r_{1}$ and $r_{0}$ are calculated from (8) as:

$r_{2}=3\left(\alpha_{1} \beta_{1}+\left(1-\alpha_{1}\right) \beta_{2}\right)$,

$r_{1}=3\left(\alpha_{1} \beta_{1}^{2}+\left(1-\alpha_{1}\right) \beta_{2}^{2}\right)$,

$r_{0}=\alpha_{1} \beta_{1}^{3}+\left(1-\alpha_{1}\right) \beta_{2}^{3}$.
By selecting $\alpha_{1}=\alpha_{2}=0.5$ and $\beta_{2}=-\beta_{1}$, we have $r_{2}=r_{0}=0$ and $r_{1}=3 \beta_{1}^{2}$. Note that in this case we have $r_{1}>0$, hence we cannot modify the parameters of $q(s)$ arbitrarily.

Let us compare the effect of dither with the effect of a possible output feedback scheme. Since the effect of dither is to change the nonlinearity $n(\cdot)$ to $n_{r}(\cdot)$ given by (4), obviously if an arbitrary nonlinear output feedback is allowed, then the same effect may be obtained. However, such an output feedback requires output measurement, which may contain measurement errors, a nonlinear operation, which may complicate the realization of the controller and may amplify the errors, and a feedback path, which may also complicate the realization. On the other hand, application of dither, if physically possible, is a much simpler scheme and does not require any measurement. Obviously a linear feedback may not produce the same effect, since it does not contain higher order terms, see (7).

\section{Simulation results}

In the simulations, we choose the dither as given by (3) with $l=2$. The parameters $\alpha_{1}, \beta_{1}, \beta_{2}$ will be calculated in each example. The period $T$ is not crucial and does not affect solutions provided that it is small, e.g. $1 / T$ is much higher than the cutoff frequency of the linear block. In all our simulations we choose $T=0.001 \mathrm{~s}$. We also note that for simulations, we use the differential equation of the form given by (9). [16].

(i). A Lur'e system with square nonlinearity: see

This system is given by $n(y)=y^{2}$ and $L(s)=$ $1 / q(s)$ with $q(s)=s^{3}+b_{2} s^{2}+b_{1} s+b_{0}$ which can be written as follows (see (2)):

$y^{(3)}+b_{2} \ddot{y}+b_{1} \dot{y}+b_{0} y+y^{2}=0$.

This system exhibits the following behaviour for the corresponding parameters:

case $i: b_{2}=0.4, b_{1}=1.18, b_{0}=1$. In this case the system exhibits chaotic behavior.

case ii: $b_{2}=0.4, b_{1}=1.18, b_{0}=0.9$. In this case the system exhibits period-2 limit cycle.

case iii: $b_{2}=0.4, b_{1}=1.18, b_{0}=0.8$. In this case the system exhibits a limit cycle. 
After applying the dither signal $d$ and a constant input $r_{0}$ as explained above, the new dynamical equation becomes:

$y^{(3)}+b_{2} \ddot{y}+b_{1} \dot{y}+b_{0} y+(y+d)^{2}=r_{0}$.

If we use the dither signal given by (3) with $l=2$, by using (10) we see that if we apply a constant input $r(t)=r_{0}$, then the closed-loop system will be the same as given by (12) with the parameter $b_{0}$ changed to $b_{0}+r_{1}$ where $r_{0}$ and $r_{1}$ are given by (10). Hence by choosing dither parameters appropriately, we could switch the behaviour of the system between the cases $i$-iii given above. We performed 3 simulations for this system. In all simulations dither is applied at $t=200 \mathrm{~s}$. We have $x_{1}=y$ and $x_{2}=\dot{y}$.

In the first simulation, to switch from the limit cycle (i.e case iii) to chaotic motion (i.e case $i$ ), we choose the dither parameters as $\alpha_{1}=\alpha_{2}=0.5, \beta_{1}$ $=0.3, \beta_{2}=-0.1$ which results in $r_{0}=0.05$, and $r_{1}=0.2$. This effectively changes $b_{0}$ from 0.8 to 1 . The results are shown in Fig. 2. In Fig. 2(b) and (c), $x_{1}-x_{2}$ graph is plotted for $115<t<200$ and for $350<t<600$, respectively. As can be seen, the system exhibits a limit cycle behaviour prior to the application of dither (case iii) and exhibits a chaotic behaviour (case $i$ ) after the application of dither.

In the second simulation, to change the chaotic behaviour (i.e case $i$ ) to period-2 limit cycle (i.e. case $i i)$, we choose the dither parameters as $\alpha_{1}=\alpha_{2}$ $=0.5, \beta_{1}=-0.2, \beta_{2}=0.1$, which results in $r_{0}=$ 0.025 and $r_{1}=-0.1$. This effectively changes $b_{0}$ from 1 to 0.9 . We observed that the system exhibits a chaotic behaviour prior to the application of dither (case $i$ ) and exhibits a period-2 limit cycle (case $i i$ ) after the application of dither. Same approach could be used to change a regular behaviour to another one. In the third simulation, to switch from limit

(a)

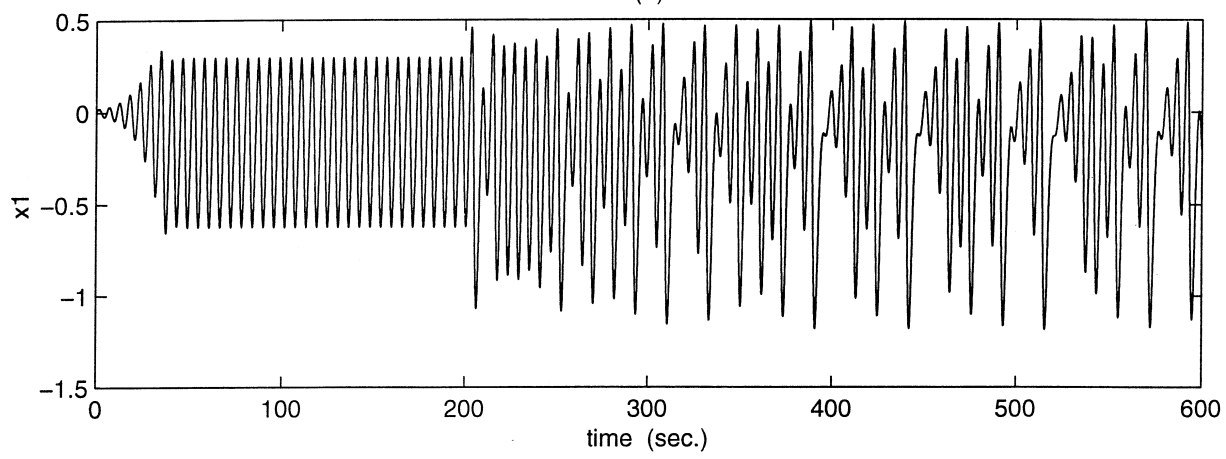

(b)

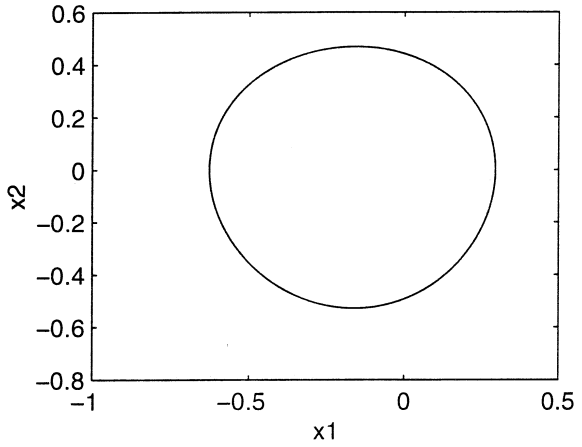

(c)

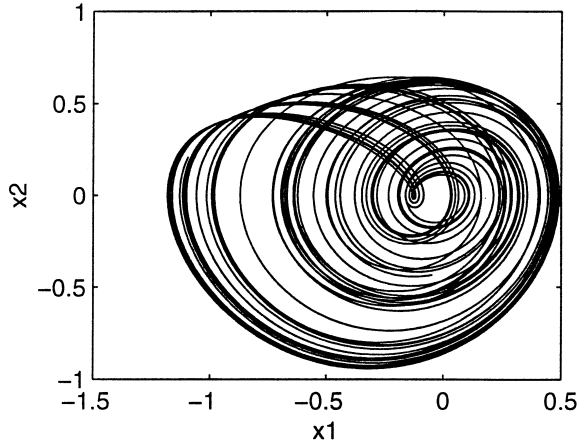

Fig. 2. Results of simulation for the system (12). Dither is applied at $t=200 \mathrm{~s}$. (a) $x_{1}=y$ versus time. (b) $x_{1}=y$ versus $x_{2}=\dot{y}$ for $115<t<200 \mathrm{~s}$. (c) $x_{1}=y$ versus $x_{2}=\dot{y}$ for $350<t<600 \mathrm{~s}$. 
cycle (i.e. case $i i i)$, to period-2 limit cycle (i.e. case ii), we choose the dither parameters as $\alpha_{1}=\alpha_{2}=$ $0.5, \beta_{1}=0.2, \beta_{2}=-0.1$ which results in $r_{0}=$ 0.025 , and $r_{1}=0.1$. The results confirm our prediction, however due to space limitation, we do not present these simulations.

(ii). Duffing Oscillator:

Duffing oscillator given below is a forced oscillator which is extensively used in nonlinear studies, since it can exhibit many types of dynamical behaviour:

$\ddot{y}+b_{1} \dot{y}+b_{0} y+p y^{3}=q \cos (\omega t)$.

This equation can be turned into Lur'e form with $L(s)=1 / q(s)$ with $q(s)=s^{2}+b_{1} s+b_{0}, n(y)=$ $p y^{3}, r(t)=q \cos \omega t$. For this system, with $p=1$, $q=11, \omega=1$, we choose the following set of parameters:

case $i: b_{1}=0.25, b_{0}=0$. For this set of parameters, the system exhibits chaotic behaviour. case ii: $b_{1}=0.25, b_{0}=0.75$. For this set of parameters, the system exhibits a limit cycle.

By choosing dither signal as given by (3) with $l=2$ and dither parameters as $\alpha_{1}=0.5, \beta_{1}=-\beta_{2}$, from (11) we obtain $r_{2}=r_{0}=0$ and $r_{1}=3 \beta_{1}^{2}$. Hence after the application of dither signal given above, resulting system is the same as given by given by (14) with $b_{0}$ changed to $b_{0}+r_{1}$. In this case if original parameters are given as in the case $i$ (i.e. chaotic behaviour), and if we choose $\beta_{1}=-\beta_{2}$ $=0.5$, then the resulting system will have the parameters as given in the case $i i$, and the resulting system exhibits a limit cycle behaviour. The results are shown in Fig. 3. In Fig. 3(b) and (c), $x_{1}-x_{2}$ graph is plotted for $0<t<200$ and for $450<t<600$, respectively. As can be seen, the system exhibits a chaotic behaviour prior to the application of dither (case $i$ ) and exhibits a limit cycle (case $i i$ ) after the application of dither. Since $r_{1}>0$, we cannot switch the behaviour given by the case $i i$ to the case $i$ by this approach.

(a)

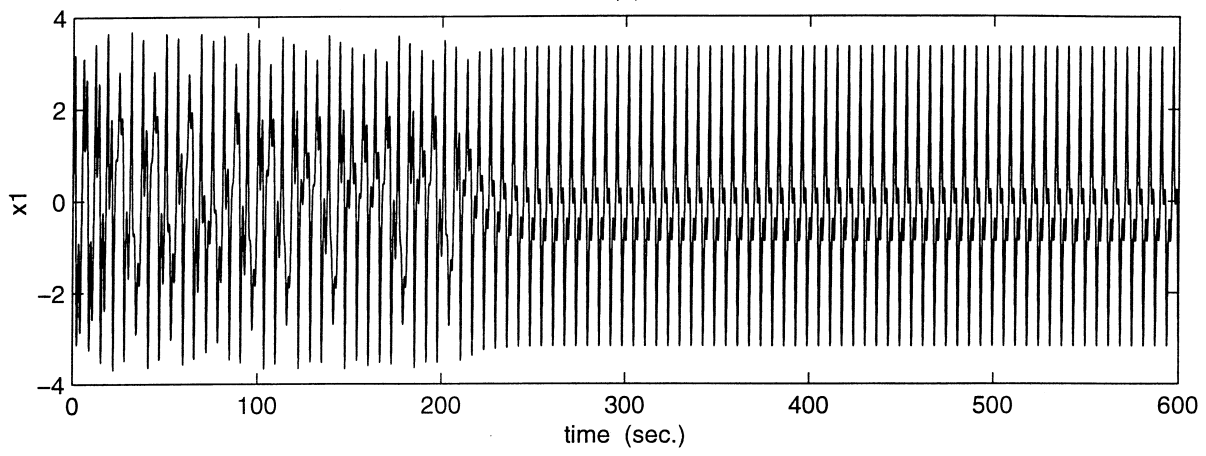

(b)

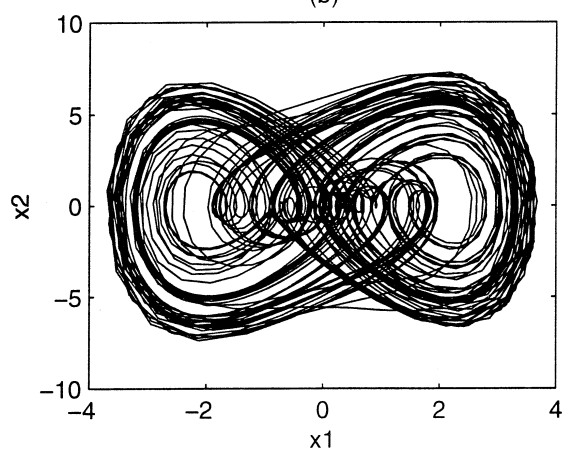

(c)

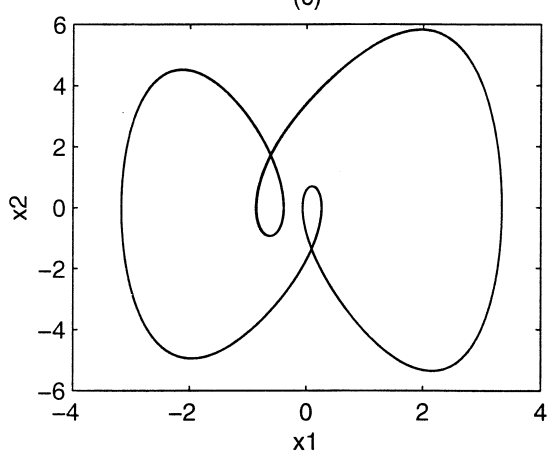

Fig. 3. Results of simulation for the Duffing oscillator (14). Dither is applied at $t=200 \mathrm{~s}$. (a) $x_{1}=y$ versus time. (b) $x_{1}=y$ versus $x_{2}=\dot{y}$ for $0<t<200 \mathrm{~s}$. (c) $x_{1}=y$ versus $x_{2}=\dot{y}$ for $450<t<600 \mathrm{~s}$. 
(iii). Van der Pol oscillator:

In [20], following version of the forced Van der Pol oscillator oscillator is considered and is shown to exhibit chaotic behaviour, see also [21]:

$\dot{x}=0.7 y+10 x\left(0.1-y^{2}\right)$,

$\dot{y}=-x+0.25 \sin (1.57 t)$.

Note that the classical forced Van der Pol equation has the form $\ddot{u}+\alpha\left(u^{2}-1\right) \dot{u}+\beta u=r(t)$ where $\alpha$ and $\beta$ are constants and $r(t)$ is the forcing term. When the forcing terms are set to zero, (15) can be put into this form. However, with the forcing term as given in (15), this is not possible. As argued in [21], this unusual type of forcing can be implemented in chemical and electronic systems. It can easily be shown that (15) cannot be put into Lur'e form. Let us assume that it is possible to apply a dither signal to the nonlinear term $y^{2}$ in (15), i.e. we may replace it by $(y+d)^{2}$. Let us assume that the same averaging is still possible, i.e. we may use (4) for equivalent nonlinearity. Note that if $d$ changes much faster then the signal $y$, which is the case if dither frequency is much larger, this averaging may make sense. Then, by using the dither signal as given by (3) with $l=2, \alpha_{1}=0.5, \beta_{1}=-\beta_{2}=\beta$, equivalent nonlinearity as given by (4) becomes $n_{r}(y)=y^{2}+$ $\beta^{2}$. By using linear analysis, it can be shown that for $\beta^{2}>0.1$, origin becomes asymptotically stable for the unforced system. Hence, by using the dither signal as given above with $\beta^{2}>0.1$, we may expect a regular (i.e. periodic behaviour) when forcing is present. In simulations, we chose $\beta=0.32$ and applied dither at $t=250 \mathrm{~s}$. The results are shown in Fig. 4. As can be seen, prior to the application of dither, the system exhibits a chaotic behaviour, whereas after the application of dither the system exhibits a regular behaviour.

(a)

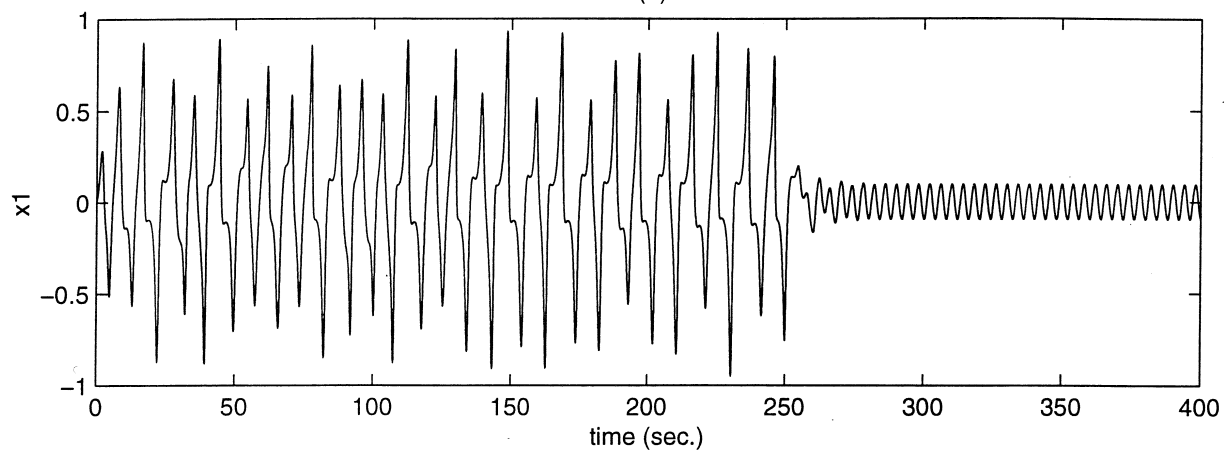

(b)

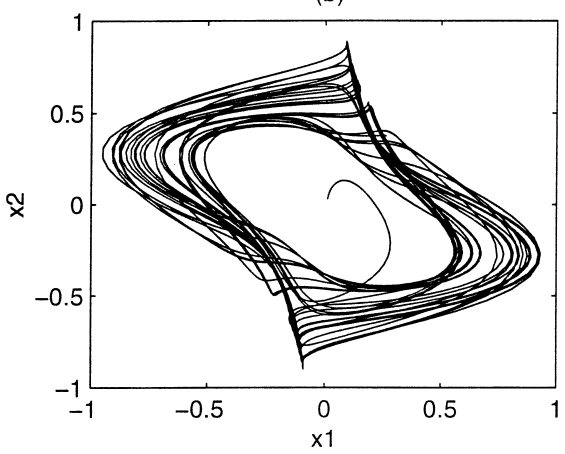

(c)

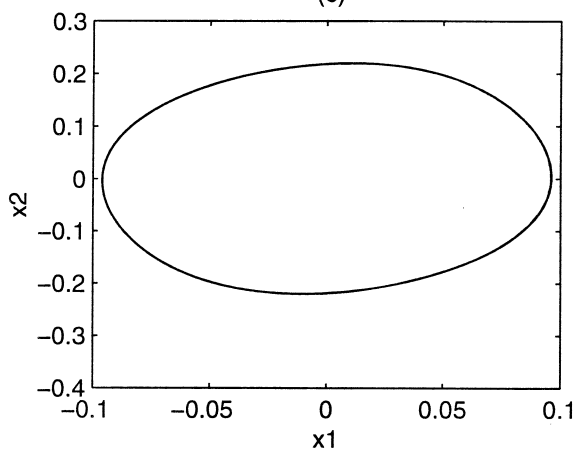

Fig. 4. Results of simulation for the Van der Pol oscillator (15). Dither is applied at $t=250 \mathrm{~s}$. (a) $x_{1}=x$ versus time. (b) $x_{1}=x$ versus $x_{2}=y$ for $0<t<250 \mathrm{~s}$. (c) $x_{1}=y$ versus $x_{2}=y$ for $300<t<400 \mathrm{~s}$. 


\section{Conclusion}

Since dither is an external signal, its application does not require any kind of measurement. Therefore main advantage of the application of dither is its simplicity. This technique is also widely used in practice in many mechanical systems, see e.g. [11,19]. In both theoretical results and practical applications reported in the literature, the system to be controlled is usually assumed to be in Lur'e form. Some well known chaotic systems and many chaotic electronic oscillators are already in this form. Especially in electronic circuits the application of dither is rather simple and effective, see [18]. In this Letter we considered three systems, one of which is not in Lur'e form, and showed that it may be possible to switch between chaotic and regular behaviour in these systems by using dither. The theory presented here justifies the use of dither for this purpose for Lur'e systems, and simulations suggest that the same theory may be extended to cover some non-Lur'e type systems as well. Whether this technique could be applied to a wider class of chaotic systems or not is an interesting question and requires further research.

\section{References}

[1] T. Shinbrot, E. Ott, C. Grebogi, J.A. Yorke, Phys. Rev. Lett. 65 (1990) 3215.
[2] E.A. Jackson, Phys. Lett. A 151 (1990) 478.

[3] E. Ott, C. Grebogi, J.A. Yorke, Phys. Rev. Lett. 64 (1990) 1196.

[4] G. Chen, X. Dong, Int'l J. of Bifur. Chaos 2 (1992) 407.

[5] G. Chen, X. Dong, Int'1 J. of Bifur. Chaos 3 (1993) 1363.

[6] T. Shinbrot, E. Ott, C. Grebogi, J.A. Yorke, Nature 363 (1993) 411.

[7] G. Chen: Control and synchronization of chaotic systems (a bibliography), ECE Dept, Univ of Houston, TX - available from ftp: ftp.egr.uh.edu/pub/TeX/chaos.tex (login name anonymous password: your email address).

[8] M. Ogorzalek, IEEE Trans. on Circ. Syst. Part 1 (1993) 40, 700 .

[9] C. Grebogi, Y.C. Lai, S. Hayes, Int'1 J. of Bifur. Chaos 7 (1997) 2175.

[10] M.A. Matias, J. Güemez, Phys. Rev. E 54 (1996) 198.

[11] D.P. Atherton, Nonlinear Control Engineering, Van Nostrand Reinhold, 1982.

[12] A.M. Steinberg, I. Kadushin, Journal of Mathematical Analysis and Applications, No. 43 (1973) 273.

[13] V.V. Alekseev, A.Y. Loskutov, Sov. Phys. Dokl. 32 (1987) 1346.

[14] R. Lima, M. Pettini, Phys. Rev. A 41 (1990) 726.

[15] Y. Braiman, I. Goldhirsch, Phys. Rev. Lett. 66 (1991) 2545.

[16] R. Genesio, A. Tesi, Automatica 28 (1992) 531.

[17] U. Ersoy, Application of dither and observer based state feedback in the control of chaotic systems, M.Sc. Thesis, Bilkent University, Ankara, Turkey, August 1996.

[18] Ö. Morgül, On the control of chaotic systems in Lur'e form by using dither, to be published in IEEE Transactions on Circuits and Systems.

[19] C.C. Fuh, P.C. Tung, Phys. Lett. A 229 (1997) 228.

[20] R. Shaw, Z. Naturf. 36a (1981) 80.

[21] J.M.T. Thompson, H.B. Stewart, Nonlinear Dynamics and Chaos, Wiley, Suffolk, 1988. 